\title{
NRF2 mitigates radiation-induced hematopoietic death
}

\author{
John P. Chute \\ Professor of Medicine, Division of Hematology/Oncology, Eli and Edythe Broad Center of Regenerative Medicine and Stem Cell Research, \\ UCLA, Los Angeles, California, USA.
}

\begin{abstract}
Fractionated, high-dose total body irradiation (TBI) is used therapeutically to myeloablate and immune suppress patients undergoing hematopoietic stem cell (HSC) transplantation. Acute exposure to ionizing radiation can have fatal effects on the hematopoietic and immune systems. Currently, therapies aimed at ameliorating ionizing radiation-associated toxicities are limited. In the February 2014 issue of the JCI, Kim and colleagues demonstrated that induction of nuclear factor erythroid 2-related factor 2 (NRF2) enhances HSC regeneration and increases survival following ionizing radiation exposure in mice. The results of this study suggest that NRF2 is a novel potential target for the development of therapeutics aimed at mitigating the toxicities of ionizing radiation exposure.
\end{abstract}

\section{Devastating events spark the origin of HSC transplantation}

The discovery that ionizing radiation disrupts the biological function of the hematopoietic and immune systems was fundamental to the advent of the fields of stem cell biology and stem cell transplantation $(1,2)$. Only in the aftermath of the atomic bombings of Hiroshima and Nagasaki were the devastating and potentially fatal hematologic effects of acute exposure to ionizing radiation fully appreciated by the medical and biological communities (1-4). In subsequent decades, landmark studies were performed by Till and McCullough $(1,2,5)$, which demonstrated that a population of cells within the murine bone marrow (BM) or spleen were capable of rescuing mice from the otherwise lethal effects of total body irradiation (TBI). In parallel, Snell (6) elucidated the role of the major histocompatibility complex in governing immune responses. Concomitantly, Thomas et al. (7) demonstrated the potential efficacy of allogeneic BM transplantation, and the field of HSC transplantation was born. Today, stem cell transplant physicians use TBI to myeloablate and immune suppress allogeneic HSC transplant patients with great success. Ironically, despite its widespread use in the preparation of patients for stem cell transplantation, few candi-

Conflict of interest: The author has declared that no conflict of interest exists.

Citation for this article: J Clin Invest. 2014; 124(3):960-961. doi:10.1172/JCI74143. date therapeutic agents capable of mitigating radiation-induced hematopoietic toxicities have been developed to date (8-12).

\section{Activation of NRF2 facilitates HSC regeneration following ionizing radiation exposure}

In the February 2014 issue of the JCI, Kim et al. describe the role of a transcription factor, nuclear factor erythroid 2-related factor 2 (NRF2), in regulating HSC function in steady-state conditions and in response to ionizing radiation (13). Kim and colleagues demonstrated that NRF2 regulates HSC function via the inducible deletion of Keap1, which encodes a cytosolic protein that inhibits NRF2 function by binding and maintaining NRF2 in the cytoplasm (14). Keap1 $1^{-/-}$mice displayed increased numbers of phenotypic HSCs and exhibited augmented HSCrepopulating capacity in a competitive repopulation assay (13). Further, Kim et al. demonstrated that mice with a constitutive deletion of Nrf2 displayed markedly increased radiosensitivity, increased hematologic toxicity, and decreased survival following TBI compared with $\mathrm{Nrf2} 2^{+/+}$mice. Importantly, induction of NRF2 provided a beneficial effect in mice following TBI. Systemic treatment of WT mice with 2-trifluoromethyl-2'methoxychalone (TMC), an oral activator of NRF2, decreased TBI-induced myelosuppression and increased the survival of irradiated mice. The mitigating effects of TMC were dependent upon NRF2 induc- tion, as TMC did not provide any benefit to $\mathrm{Nrf2^{-/- }}$ mice following TBI. Last, the mitigating effects of TMC treatment were mediated, at least in part, by an enhanced recovery of both the HSC and hematopoietic progenitor pool and mature blood cells in irradiated mice.

Given the strong effects that genetic or pharmacologic modulation of NRF2 have on the hematopoietic system in mice, it will be important to discern the precise mechanism of action through which NRF2 mediates these effects. NRF2-dependent responses may be related to its established function in mediating cytoprotection in response to ROS (10); however, Kim et al. have provided insight into alternative NRF2-mediated mechanisms of action at the levels of both cellular effects and signaling events (13). Interestingly, using a tamoxifen-inducible CMVCre model, Kim and colleagues showed that selective activation of NRF2 in BM stromal cells augmented HSC expansion in vitro (13), suggesting that NRF2 regulates HSC fate via both an HSC-intrinsic mechanism and via non-HSC autonomous effects within the BM microenvironment. Kim et al. also demonstrated that administration of TMC induced Notch receptor signaling in hematopoietic cells in vivo and that inhibition of Notch signaling via a $\gamma$ secretase inhibitor abrogated the beneficial effects of TMC on hematopoiesis. Interestingly, TMC-mediated induction of NRF2 also upregulated expression of the Notch ligand Jagged on BM stromal cells, and antibody-based blockade of Jagged abrogated TMC-mediated expansion of hematopoietic progenitor cells ex vivo. Taken together, these data suggest that activation of NRF2 in the BM microenvironment mediates the expansion of HSC/ progenitor cells via the induction of Notch signaling on target hematopoietic cells.

\section{Implications and future directions}

The results described by Kim et al. (13) suggest important therapeutic implications regarding NRF2 signaling in 
hematopoiesis. First, it may be possible to induce human HSC expansion in vitro or in vivo via pharmacologic activation of NRF2. If successful, such capability could have a significant impact on the field of HSC transplantation. Second, pharmacologic induction of NRF2 could serve to mitigate the potentially fatal effects of radiation-induced hematopoietic failure in victims of acute radiation sickness. This latter possibility has major public health implications, given the ongoing worldwide threat of radiological or improvised nuclear devices as weapons of terrorism (15). Commensurate with this, the development of effective therapies to mitigate acute radiation sickness is a high priority for the US federal government (16).

In summary, the study by Kim et al. (13) provides new insights into the fundamental role of NRF2 in regulating the hematopoietic response to ionizing radiation. Of equal importance, NRF2 represents a new mechanistic target for the development of therapeutics to accelerate hematopoietic reconstitution both in victims of acute radiation sickness and patients undergoing stem cell transplantation.

Address correspondence to: John P. Chute, Division of Hematology/Oncology, Room 545, Orthopedic Hospital Research Center, UCLA, Los Angeles, California 90095, USA. Phone: 310.206.3037; E-mail: JChute@mednet.ucla.edu.

\footnotetext{
1. Till J, McCulloch E. A direct measurement of the radiation sensitivity of normal mouse bone marrow cells. Radiat Res. 2012;178(2):AV3-AV7.

2. McCulloch E, Till J. The radiation sensitivity of normal mouse bone marrow cells, determined by quantitative marrow transplantation into irradiated mice. Radiat Res. 1960;13:115-125.

3. Okita T. Review of thirty years study of Hiroshima and Nagasaki atomic bomb survivors. II. Biological effects. A. Acute effects. J Radiat Res. 1975;16(suppl):49-66.

4. Hiroshima Day Committee. Hiroshima and Nagasaki Bombing: Facts about the atomic bomb. Hiroshima Day Committee Web site. http://www. hiroshimacommittee.org/Facts_NagasakiAndHiro shimaBombing.htm. Accessed January 24, 2014.

5. Till J, McCulloch E, Siminovitch L. A stochastic model of stem cell proliferation, based on the growth of spleen colony forming cells. Proc Natl Acad Sci U S A. 1964;51:29-36.

6 . Snell GD. Methods for the study of histocompatibility genes. J Genet. 1948;49(2):87-108.

7. Thomas ED, Lochte HL Jr, Ferrebee JW. Irradiation
}

of the entire body and marrow transplantation: some observations and comments. Blood. 1959;14(1):1-23.

8. Farese AM, et al. Filgrastim improves survival in lethally irradiated nonhuman primates. Radiat Res. 2013;179(1):89-100.

9. Guinan E, et al. Bactericidal/permeability-increasing protein (rBPI21) and fluoroquinolone mitigate radiation-induced bone marrow aplasia and death. Sci Transl Med. 2011;3(110):110ra-118.

10. Burdelya L, et al. An agonist of toll-like receptor 5 has radioprotective activity in mouse and primate models. Science. 2008;320(5873):226-230.

11. Himburg $\mathrm{H}$, et al. Pleiotrophin regulates the expansion and regeneration of hematopoietic stem cells. Nat Med. 2010;16(4):475-482.

12. Doan PL, et al. Epidermal growth factor regulates hematopoietic regeneration after radiation injury. Nat Med. 2013;19(3):295-304.

13. Kim J-H, et al. NRF2-mediated Notch pathway activation enhances hematopoietic reconstitution following myelosuppressive radiation. J Clin Invest. 2014;124(2):730-741

14. Kensler TW, Wakabayashi N, Biswal S. Cell survival responses to environmental stresses via the Keap1Nrf2-ARE pathway. Annu Rev Pharmacol Toxicol. 2007;47:89-116.

15. Kim SB, et al. Targeting of Nrf2 induces DNA damage signaling and protects colonic epithelial cells from ionizing radiation. Proc Natl Acad Sci U S A. 2012;109(43):E2949-E2955.

16. Weinstock DM, et al. Radiologic and nuclear events: contingency planning for hematologists/ oncologists. Blood. 2008;111(12):5440-5445.

17. Hafer N, et al. NIAID/NIH radiation/nuclear countermeasures product research and development program. Health Phys. 2010;98(6):903-905.

\title{
Fanning the flames to regenerate the heart
}

\author{
Paul R. Riley
}

Department of Physiology, Anatomy and Genetics, University of Oxford, Oxford, United Kingdom.

\begin{abstract}
Damage to the adult mammalian heart is irreversible, and lost cells are not replaced through regeneration. In neonatal mice, prior to $\mathrm{P} 7$, heart tissue can be regenerated after injury; however, the factors that facilitate cardiac regeneration in the neonatal heart are not known. In this issue of the JCI, Aurora and colleagues evaluated the immune response following myocardial infarction in P1 mice compared with that in P14 mice, which have lost their regenerative capacity, and identified a population of macrophages as mediators of cardiac repair. Further understanding of the immune modulators that promote the regenerative properties of this macrophage subset could potentially be exploited to recapitulate regenerative function in the adult heart.
\end{abstract}

\section{Repair without regeneration in the damaged adult heart}

Myocardial infarction (MI), commonly known as heart attack, results in the loss of around 1 billion heart muscle cells and destruction of surrounding blood vessels. Once damaged, adult heart cells cannot

Conflict of interest: The author has declared that no conflict of interest exists.

Citation for this article: $J$ Clin Invest. 2014;

124(3):961-964. doi:10.1172/JCI74418. be replaced through regeneration; therefore, an alternate form of wound healing is orchestrated by the immune system. Inflammatory cells migrate to the injured heart, ensuring the clearance of harmful cell debris and repair of the damaged area via formation of a fibrotic scar. The MI-associated inflammatory response is largely stereotypical and considered to be a function of the innate immune system. While inflammatory tissue reparation is initially beneficial, the scarring leads to pathological remodeling of the heart and compromised cardiac function over time. Moreover, if the initial acute inflammatory response becomes chronic, host tissue will be subject to continued damage.

Efforts to promote heart regeneration have largely centered on mechanisms to replace lost cardiovascular cells via cell transplantation or through stimulation of resident cells, such as altering progenitor cell differentiation or inducing muscle cell division. Relatively little attention has been paid to the local environment that transplanted or newly stimulated cells encounter in the heart following MI, which constitutes a proinflammatory milieu, much of which is cytotoxic, and an ensuing fibrosis that disrupts essential matrix. Together, the proinflammatory environment along with a dysfunctional matrix prevent engraftment and integration of new cells into survived heart tissue. While the default immune response to MI appears to ensure a quick fix of the heart, it precludes cell replacement and tissue regeneration. 